\title{
Universal doping dependence of the ground-state staggered magnetization of cuprate superconductors
}

\author{
Rinat Ofer, ${ }^{1}$ Amit Keren, ${ }^{1}$ Omar Chmaissem, ${ }^{2}$ and Alex Amato ${ }^{3}$ \\ ${ }^{1}$ Physics Department, Technion-Israel Institute of Technology, Haifa 32000, Israel \\ ${ }^{2}$ Materials Science Division, Argonne National Laboratory, 9700 South Cass Avenue, Argonne, Illinois 60439, USA \\ and Physics Department, Northern Illinois University, DeKalb, Illinois 60115, USA \\ ${ }^{3}$ Paul Scherrer Institute, CH 5232 Villigen PSI, Switzerland \\ (Received 10 August 2008; published 10 October 2008)
}

\begin{abstract}
Using muon spin rotation we determine the zero-temperature staggered antiferromagnetic order parameter $M_{0}$ versus hole doping measured from optimum $\Delta p_{m}$, in the $\left(\mathrm{Ca}_{x} \mathrm{La}_{1-x}\right)\left(\mathrm{Ba}_{1.75-x} \mathrm{La}_{0.25+x}\right) \mathrm{Cu}_{3} \mathrm{O}_{y}$ system. In this system the maximum $T_{c}$ and the superexchange $J$ vary by $30 \%$ between families $(x) . M_{0}\left(x, \Delta p_{m}\right)$ is found to be $x$ independent. Using neutron diffraction we also determine the lattice parameters variations for all $x$ and doping. The oxygen buckling angle is found to change with $x$, implying a change in the holes kinetic energy. We discuss the surprising insensitivity of $M_{0}\left(x, \Delta p_{m}\right)$ to the kinetic-energy variations in the framework of the $t$ - $J$ model.
\end{abstract}

DOI: $10.1103 /$ PhysRevB.78.140508

It is widely agreed that the antiferromagnetic (AFM) phase of the cuprates should be addressed as a doped Mott insulator, where holes are moving on a two-dimensional (2D) AFM background. ${ }^{1}$ This scenario is described by the $t-J$ model Hamiltonian where $t$ and $t^{\prime}$ are the near and next-near neighbor hoppings, respectively, and $J$ is the Heisenberg superexchange. Above some critical doping the zero-temperature staggered AFM order parameter $M_{0}$ is destroyed, and the cuprates enter a glassy, phase separated, state. Since the glassy state precedes superconductivity, understanding this transition is crucial to understanding the cuprates. Particularly interesting is the doping dependence of $M_{0}$ and its variations with the different energy scales. These variations were calculated theoretically ${ }^{2}$ but not measured in a controlled manner. Such measurements could shed light on the effective Hamiltonian governing the holes at $T \rightarrow 0$ in the underdoped region. While $J$ can be measured relatively simply with neutron or Raman scattering on a single crystal, it is very difficult to determine $t$ experimentally.

In this work we determine $t / J$ from their lattice-parameter dependence, including the buckling angle, for different cuprate families $(x)$ of the $\left(\mathrm{Ca}_{x} \mathrm{La}_{1-x}\right)\left(\mathrm{Ba}_{1.75-x} \mathrm{La}_{0.25+x}\right) \mathrm{Cu}_{3} \mathrm{O}_{y}$ (CLBLCO) system, where the maximum $T_{c}\left(T_{c}^{\mathrm{max}}\right)$ and $J$ varies by about $30 \%$ between families. ${ }^{3}$ This is done by Rietvelt refinement of neutron diffraction. We also determine the doping dependence of $M_{0}$, which is expected to depend on $t / J$. Zero field muon spin rotation ( $\mu \mathrm{SR})$ is employed for this purpose. Our main finding is that the doping dependence of $M_{0}$ is universal despite the fact that $t / J$ varies between families.

We chose to work with the CLBLCO system for several reasons. This is a high-temperature superconductor (HTSC) with $\mathrm{YBa}_{2} \mathrm{Cu}_{3} \mathrm{O}_{y}$ (YBCO) structure. The family index $x$ varies in the range $0.1 \leq x \leq 0.4$. Doping is possible all the way to the overdoped regime. All compounds are tetragonal and there is no chain ordering as in YBCO. ${ }^{4}$ As we show below, there are minimal structural differences between the families. In addition, the level of disorder as detected by $\mathrm{Ca}$ nuclear magnetic resonance (NMR) (Ref. 5) and $\mathrm{Cu}$ nuclear quadrupole resonance (NQR) (Ref. 6) is identical for the different families. The phase diagram is presented in Fig. 1(b) showing the antiferromagnetic Néel temperature $T_{N}$, the spin-glass temperature $T_{g}$ where islands of spins freeze, and the superconducting critical temperature $T_{c}$; note the axis breaker. In this phase diagram $T_{N}$ (Ref. 3) and $T_{g}$ (Ref. 7) were measured by $\mu \mathrm{SR}$, and $T_{c}$ was measured by resistivity. ${ }^{4}$ The spinglass phase penetrates into the superconducting phase. It also slightly penetrates into the Néel phase in the sense that a first transition, to long-range order, takes place near $200 \mathrm{~K}$, and a second transition, with additional spontaneous fields, takes place near $10 \mathrm{~K}$. Each transition is a continuous function of doping.

The neutron powder-diffraction experiments were performed at the special environment powder diffractometer at Argonne's Intense Pulsed Neutron Source (see Ref. 8 for

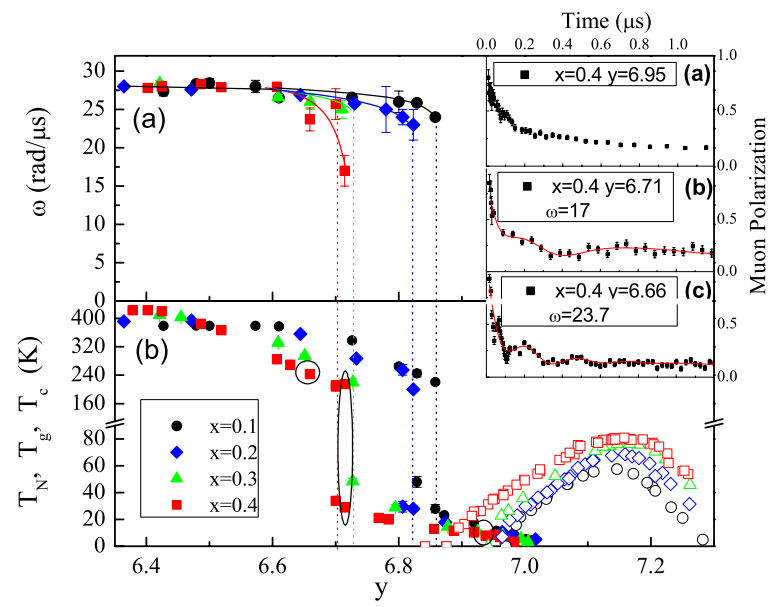

FIG. 1. (Color online) (a) The zero-temperature muon oscillation angular frequency $\omega$ as a function of the chemical doping $y$ for all four of the $\left(\mathrm{Ca}_{x} \mathrm{La}_{1-x}\right)\left(\mathrm{Ba}_{1.75-x} \mathrm{La}_{0.25+x}\right) \mathrm{Cu}_{3} \mathrm{O}_{y}$ (CLBLCO) families. The antiferromagnetic zero-temperature order parameter $M_{0}$ is proportional to $\omega$. (b) The CLBLCO phase diagram from Ref. 3 including magnetic (closed symbols) and superconducting (open symbols) critical temperatures. The insets show raw muon polarization data and fits to Eq. (3) for three samples from the $x=0.4$ family marked in panel (b) 


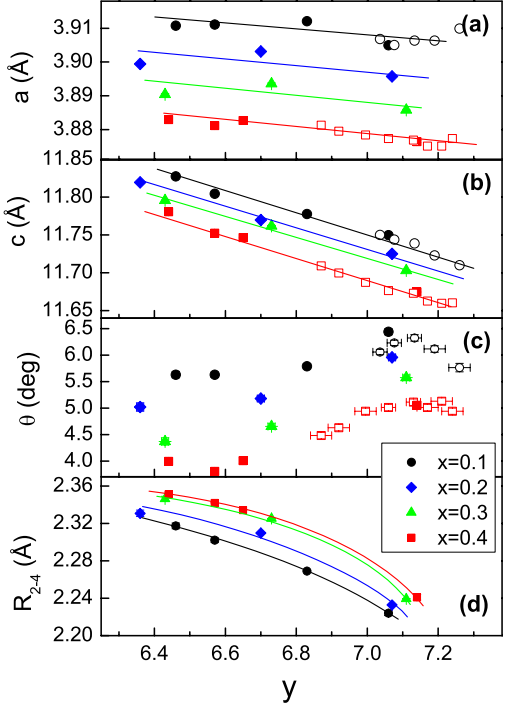

FIG. 2. (Color online) The parameters extracted from a neutrondiffraction experiment as a function of oxygen doping for the four families of CLBLCO. (a) The lattice parameter $a$. (b) The lattice parameter $c$. (c) $\theta$-the buckling angle between the copper and oxygen in the plane. (d) $R_{24}$-the distance between the in-plane copper and the apical oxygen. The error bars are smaller than the symbols. The empty symbols are measurements taken from Ref. 8. The lines are guides to the eye.

more details). Figure 2 shows a summary of the lattice parameters. The empty symbols represent data taken from Ref. 8. All the parameters are family dependent, however, not to the same extent. The lattice parameters $a$ and $c$, depicted in Figs. 2(a) and 2(b), change by up to about $0.5 \%$ between the two extreme families $(x=0.1$ and $x=0.4)$. The in-plane $\mathrm{Cu}$ $\mathrm{O}-\mathrm{Cu}$ buckling angle is shown in Fig. 2(c). This angle is nonzero since the oxygen is slightly out of the $\mathrm{Cu}$ plane and closer to the $Y$ site of the YBCO structure. The buckling angle shows strong variation between the families; there is about a $30 \%$ change from the $x=0.1$ family to $x=0.4$. This change is expected since as $x$ increases, a positive charge is moving from the $Y$ to the $\mathrm{Ba}$ site of the YBCO structure, pulling the oxygen toward the plane and flattening the $\mathrm{Cu}-$ $\mathrm{O}-\mathrm{Cu}$ bond.

We believe that this property is the main cause for the different $J$ and therefore different $T_{c}^{\max }$ between the CLBLCO families. ${ }^{3}$ Nevertheless, we note that Pavarini et $a l .{ }^{9}$ showed that in many cuprates families $T_{c}^{\text {max }}$ scales with $t^{\prime} / t$. $t^{\prime}$ is controlled by the hybridization of the $\mathrm{Cu} 4 s$ with the apical oxygen $2 p_{z}$, hence $T_{c}^{\max }$ scales with the distance $R_{24}$ between the in-plane copper and the apical oxygen. In Fig. 2(d) we show $R_{24}$ for our CLBLCO samples. Our results also support qualitatively Pavarini's conclusion.

From the lattice parameters and the buckling angle it is possible to construct the $t / J$ ratio assuming that the Hubbard $U$ and the charge-transfer energy $\Delta$ are family independent. The basic quantity is the hopping integral $t_{p d}$ between a $\mathrm{Cu} 3 d_{x^{2}-y^{2}}$ and $\mathrm{O} 2 p .^{10}$ This hopping integral is proportional to bond length to the power $-3.5 .^{11}$ The hopping from the $\mathrm{O} 2 p$ to the next $\mathrm{Cu} 3 d_{x^{2}-y^{2}}$ involves again the bond length and cosine of the angle. Thus, the $\mathrm{Cu}$ to $\mathrm{Cu}$ hopping depends on $a$ and $\theta$ as

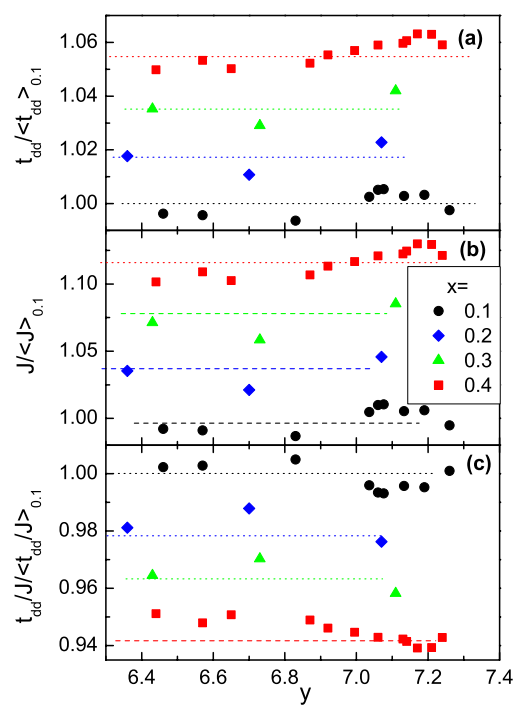

FIG. 3. (Color online) (a) The hopping rate $t_{d d}$, and (b) the superexchange coupling $J$ calculated from Eqs. (1) and (2), using the parameters shown in Fig. 2. (c) The ratio $t_{d d} / J$ as a function of doping. The dotted lines are guides to the eye. All data sets are normalized to the $x=0.1$ family. The error bars are smaller than the symbols.

$$
t_{d d} \propto \frac{\cos \theta}{a^{7}} .
$$

$J$ is proportional to $t_{d d}^{2}$, hence,

$$
J \propto \frac{\cos ^{2} \theta}{a^{14}} .
$$

Estimates of the $t_{d d}$ and $J$, normalized to the averaged values of the $x=0.1$ family, $\left\langle t_{d d}\right\rangle_{0.1}$ and $\langle J\rangle_{0.1}$ are presented in Figs. $3(\mathrm{a})$ and 3(b). Although there is a variation in $t$ and $J$ within each family, the variation is much larger between the families. $J$ increases with increasing $x$, in qualitative agreement with experimental determination of $J .^{3}$ In Fig. 3(c) we show the normalized ratio $t / J$ for the four CLBLCO families. There is about $5 \%$ difference between the two extreme families. But we stress that this determination of $t / J$ is only an estimate, used in practice to set the oxygen level spacing between samples in the $\mu$ SR experiment. More accurate calculations of $t / J$ are in progress and preliminary data indicate that $t / J$ varies by more than $10 \%$ between families. ${ }^{12}$

Next we determine the doping dependence of the order parameter using zero field $\mu \mathrm{SR}$. The experiments were done on the GPS beam line at the Paul Scherrer Institute, Switzerland. The muon oscillation angular frequency $\omega$ is proportional to the local magnetic field it experiences. Therefore, it can be used to determine the staggered magnetization $M$. Typical muon polarization curves at $T=5 \mathrm{~K}$ are presented in the insets of Fig. 1 for three samples from the $x=0.4$ family on the border between Néel and glass order. These three samples are marked on the phase diagram in Fig. 1(b). More raw data can be found in Ref. 3. The sample in inset (a) is in the spin-glass phase; it has no long-range magnetic order and hence has no oscillations. The sample in inset (c) is in the 
antiferromagnetic phase, and so it has strong oscillations at low temperatures. Finally, the sample in inset (b) is an example of an intermediate sample and thus has weaker oscillations. tion

The best fit of the polarization is achieved with the func-

$$
P(t)=\sum_{i=1}^{3} A_{i} \exp \left(-\lambda_{i} t\right) \cos \left(\omega_{i} t\right)
$$

with $\omega_{3}=0$; the fit is shown in insets (b) and (c) of Fig. 1 by the solid line. The reason for multiple frequencies is that the muons stop at different sites in the unit cell. The order parameter extracted from the high angular frequency, around a few tens of radians per micro-second is known to agree with neutron-scattering experiments. ${ }^{13}$ The lower angular frequency is believed to emerge from metastable muon sites and is not used for further analysis.

The muon polarization was measured at low temperatures, typically from 5 to $200 \mathrm{~K}$, and the oscillation for $T \rightarrow 0$ was extracted from extrapolation. Figure 1(a) shows a summary of the oscillation angular frequency $\omega(T \rightarrow 0)$ as a function of the chemical doping $y$ for all four CLBLCO families. In this plot the AFM critical doping, where the oscillations disappear, is different for each family. Not surprisingly, this is the same oxygen doping where the Néel order is replaced by the spin-glass phase in the diagram. However, the chemical doping is different from the mobile hole doping $p_{m}$, and a rescaling of the doping axis is required.

The scaling is designed so that the $T_{c}$ domes of all families, normalized by $T_{c}^{\mathrm{max}}$, will collapse on to a single dome. For this purpose the mobile hole parameter measured from optimum $\Delta p_{m}$ is defined by $\Delta p_{m}=K(x) \cdot\left(y-y_{\max }\right)$, where $y_{\max }$ is the optimal oxygen doping, and $K(x)$ is a familydependent scaling parameter. $K$ should be thought of as doping efficiency parameter connecting oxygen level to mobile holes in the $\mathrm{CuO}_{2}$ planes. The best scaling was found using $K=0.76,0.67,0.54,0.47$ for $x=0.1, \ldots, 0.4$, respectively, and is shown in Fig. 4(a). The errors of $K$ are discussed below. Despite the fact that $K(x)$ was chosen to scale the $T_{c}$ domes, by the same token other critical temperatures scale as well. Figure 4(a) also shows, $T_{g} / T_{c}^{\mathrm{max}}$ and $T_{N} / T_{c}^{\mathrm{max}}$, for all families, as a function of $\Delta p_{m}$. The $T_{g} / T_{c}^{\max }$ curves of all families collapse onto each other and $T_{N} / T_{c}^{\max }$ for the $x=0.2-0.4$ families collapse onto each other. The reason $T_{N}$ of the $x$ $=0.1$ family is not in line with the others is due to interactions between planes.

The Néel temperature stems from the three-dimensional (3D) interaction, and it is a function of not only the in-plane $J$, but also interplane couplings $J_{\perp}$ and other anisotropies. In the Heisenberg model $T_{N}=J t_{N}\left(\alpha_{\text {eff }}\right)$ where the effective anisotropy $\alpha_{\text {eff }}$ is mainly set by $J_{\perp} / J$, and $t_{N}\left(\alpha_{\text {eff }}\right)$ is a known logarithmic function of the anisotropy. ${ }^{14}$ We determine $\alpha_{\text {eff }}$ and extract $J$ from $T_{N}$. This is done by measuring the temperature dependence of the muon rotation angular frequency $\omega(T)$, which is proportional to order parameter $M(T)$. The

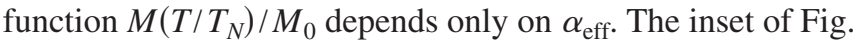
4(a) shows $\omega\left(T / T_{N}\right) / \omega(T \rightarrow 0)$ for two samples marked in Fig. 4(a), and a fit to the predicted behavior given in Refs. 3

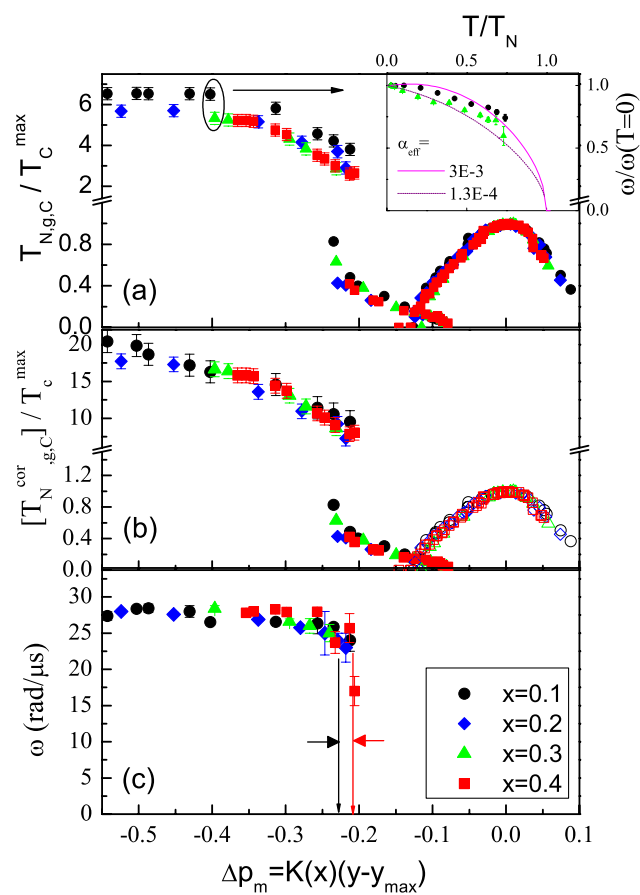

FIG. 4. (Color online) (a) The CLBLCO phase diagram after rescaling; for each family $(x)$, the critical temperatures are normalized by $T_{c}$ at optimal doping, and $y$ is replaced by $\Delta p_{m}$ (see text for details). (b) The same as (a) but the Néel temperature is corrected for anisotropy contribution so it is the same as $J$ for the parent compound (see text). (c) The zero-temperature muon oscillation angular frequency as a function of $\Delta p_{m}$ for all four CLBLCO families; an equivalent to the staggered magnetization $M_{0}$ versus mobile hole density. The arrows show the expected variation of the critical doping from a $5 \%$ variation in $t / J$.

and 14. The $x=0.1$ sample clearly has a bigger $\alpha_{\text {eff }}$ than the $x=0.3$ sample, and is more $3 \mathrm{D}$-like. Using this method we determined $\alpha_{\text {eff }}$ for all samples with Néel order and defined the quantity $T_{N}^{\text {cor }}=T_{N} / t_{N}\left(\alpha_{\text {eff }}\right)$ for these samples. ${ }^{3}$ For zero doping $T_{N}^{\text {cor }}=J$. When the system is doped, $T_{N}$ is also affected by hopping and $T_{N}^{\mathrm{cor}}=J$ is no longer valid. $T_{N}^{\text {cor }}$ replaces $T_{N}$ in Fig. 4(b) which otherwise is the same as Fig. 4(a). After this replacement the entire phase diagram scales to a single unified curve, indicating that $T_{c}^{\max } \propto J$ and that a single energy scale controls magnetism and superconductivity.

Figure 4(c) shows $\omega(T \rightarrow 0)$ as a function of $\Delta p_{m}$ for each family. The scalability of the phase diagram, as explained above, suggests that $\Delta p_{m}$ is a parameter proportional to the mobile hole density variation. Hence Fig. 4(a) is equivalent to a plot of the AFM order parameter at zero temperature as a function of mobile hole density. This plot shows that the order parameter is universal for all families, and in particular the AFM critical doping is family independent. To demonstrate this point we show, using the two arrows in Fig. 4(c), what should have been the difference in the critical doping had it been proportional to $t / J$, and changed between the $x$ $=0.4$ and $x=0.1$ by $5 \%$ (of 0.3 ) as indicated in Fig. 3(c). Thus, we conclude that $M_{0}\left(x, \Delta p_{m}\right)$ is $x$ independent, hence independent of $t / J$.

The above conclusion could, a priori, depend on the choice of the $K$ 's and $y_{\max }$. A different set of $K$ 's or $y_{\max }$ 
would shift the magnetic critical doping with respect to each other. However, it will also shift the normalized $T_{c}$ domes, $T_{g}$ line, and $T_{N}^{\text {cor }}$ line with respect to each other. We have attempted to use a different set of $K$ 's and $y_{\max }$ 's, which will not noticeably destroy the scaling of the normalized critical temperatures. We could not generate a variation of more than $2 \%$ in the $M_{0}$ critical doping. In other words, the different sets of $K$ 's and $y_{\max }$ 's always kept the critical doping well between the two vertical arrows in Fig. 4(c).

This surprising result could be discussed using three scenarios: (I) That different values of $t / J$ correspond to different values of $K$, namely, the changes in $t / J$ are canceled out by the rescaled doping axis. However, there is no theoretical backing for this scenario. (II) It may be that at low temperatures the effective Hamiltonian is given by a $t$ - $J$ model but with an effective $t$ that is proportional to $J$. Indeed, there are indications that for small hole doping in an antiferromagnet the bandwidth of the hole dispersion is set by $J$ (instead of t). ${ }^{15}$ However, the spatial size of each hole quasiparticle (spin polaron), and thus the critical doping, does depend on $t / J$. Moreover, it is not clear how spin polarons destroy the AFM order and at which doping. (III) An alternative explanation is that the destruction of the AFM order parameter is not a result of single holes hopping and should be described by a completely different effective Hamiltonian; perhaps hopping of boson pairs. ${ }^{16}$ In this case $t$ should be absorbed into the creation of tightly bound bosons leaving a prominent energy scale $J$. The proximity of the magnetic critical doping to superconductivity makes this possibility appealing.

The last possibility could also solve a profound riddle in the study of the CLBLCO system. This system was found to obey the Uemura relation $T_{c} \propto n_{s},{ }^{17}$ where $n_{s}$ is the superconducting carrier density, in both underdoped and overdoped regions. ${ }^{18}$ At the same time $T_{c}^{\max }$ scales with $J$ as indicated before. Therefore, the Uemura relation should be rewritten as $T_{c} \propto J n_{s}$. What is then the role of $t$ ? Our finding that the magnetic order parameter versus doping is universal suggests that even before superconductivity appears, $t$ becomes less relevant. This suggestion does have theoretical support. ${ }^{19}$

To conclude, an estimate of $t$ and $J$ from simple structure considerations using neutron diffraction shows that the origin of the different energy scales between the CLBLCO families is mainly the different buckling angles. The difference in $t / J$ between the two extreme families is about $5 \%$. Although this is not an accurate way to measure the hopping rate or superexchange coupling, it does set the scale for the expected variation in the AFM critical doping. Using $\mu \mathrm{SR}$, the AFM order parameter as a function of oxygen was determined for different families of the CLBLCO system. We used a scaling transformation to move from oxygenation level to mobile holes. Our measurements show that, at zero temperature, the order parameter as a function of mobile holes is independent of $t / J$ within the required accuracy.

We would like to thank D.-G. A. Sawatzky, M.-B. Lepetit, A. Auerbach, and E. Amit for very helpful discussions. We acknowledge financial support from the Israel Science Foundation, the European Commission under the 6th Framework Programme, and the Posnansky research fund in hightemperature superconductivity. We are also grateful to the PSI facilities for high quality muon beams and technical support. This work was partially supported by the Division of Materials Sciences and Engineering Division of the Office of Basic Energy Sciences, U.S. Department of Energy Office of Science, under Contract No. DE-AC02-06CH11357, and through the Key Action: Strengthening the European Research Area, Research Infrastructures Contract No. RII3-CT2003-505925.
${ }^{1}$ E. Dagotto, Rev. Mod. Phys. 66, 763 (1994).

${ }^{2}$ G. Khaliullin and P. Horsch, Phys. Rev. B 47, 463 (1993); A. Belkasri and J. L. Richard, ibid. 50, 12896 (1994); J. L. Richard and V. Yu. Yushankhaï, ibid. 50, 12927 (1994); D. Yamamoto and S. Kurihara, ibid. 75, 134520 (2007).

${ }^{3}$ R. Ofer, G. Bazalitsky, A. Kanigel, A. Keren, A. Auerbach, J. S. Lord, and A. Amato, Phys. Rev. B 74, 220508(R) (2006).

${ }^{4}$ D. Goldschmidt, G. M. Reisner, Y. Direktovitch, A. Knizhnik, E. Gartstein, G. Kimmel, and Y. Eckstein, Phys. Rev. B 48, 532 (1993).

${ }^{5}$ Sebastien Marchand, Ph.D. thesis, Université Paris 6, 2005.

${ }^{6}$ A. Keren, A. Kanigel, and G. Bazalitsky, Phys. Rev. B 74, 172506 (2006).

${ }^{7}$ A. Kanigel, A. Keren, Y. Eckstein, A. Knizhnik, J. S. Lord, and A. Amato, Phys. Rev. Lett. 88, 137003 (2002).

${ }^{8}$ O. Chmaissem, J. D. Jorgensen, S. Short, A. Knizhnik, Y. Eckstein, and H. Shaked, Nature (London) 397, 45 (1999).

${ }^{9}$ E. Pavarini, I. Dasgupta, T. Saha-Dasgupta, O. Jepsen, and O. K. Andersen, Phys. Rev. Lett. 87, 047003 (2001).

${ }^{10}$ J. Zaaneann and D. G. A. Sawatzky, Can. J. Phys. 65, 1262 (1987).

${ }^{11}$ W. A. Harrison, Electronic Structure and the Properties of Solids
(Freeman, San Francisco, 1980).

${ }^{12} \mathrm{~S}$. Petit and M.-B. Lepetit (unpublished).

${ }^{13}$ A. Keren, L. P. Le, G. M. Luke, B. J. Sternlieb, W. D. Wu, Y. J. Uemura, S. Tajima, and S. Uchida, Phys. Rev. B 48, 12926 (1993).

${ }^{14}$ D. P. Arovas and A. Auerbach, Phys. Rev. B 38, 316 (1988); B. Keimer, A. Aharony, A. Auerbach, R. J. Birgeneau, A. Cassanho, Y. Endoh, R. W. Erwin, M. A. Kastner, and G. Shirane, ibid. 45, 7430 (1992).

${ }^{15}$ S. Schmitt-Rink, C. M. Varma, and A. E. Ruckenstein, Phys. Rev. Lett. 60, 2793 (1988); C. L. Kane, P. A. Lee, and N. Read, Phys. Rev. B 39, 6880 (1989); Z. Liu and E. Manousakis, ibid. 45, 2425 (1992); E. Altman and A. Auerbach, ibid. 65, 104508 (2002).

${ }^{16}$ M. Havilio and A. Auerbach, Phys. Rev. Lett. 83, 4848 (1999).

${ }^{17}$ Y. J. Uemura et al., Phys. Rev. Lett. 62, 2317 (1989).

${ }^{18}$ A. Keren, A. Kanigel, J. S. Lord, and A. Amato, Solid State Commun. 126, 39 (2003).

${ }^{19}$ S. S. Kancharla, B. Kyung, D. Sénéchal, M. Civelli, M. Capone, G. Kotliar, and A.-M. S. Tremblay, Phys. Rev. B 77, 184516 (2008). 